\title{
Metasurface-Assisted Quantum Ghost Discrimination of Polarization Objects
}

\author{
Andres Vega, ${ }^{1, *}$ Thomas Pertsch, ${ }^{1,2}$ Frank Setzpfandt, ${ }^{1}$ and Andrey A. Sukhorukov ${ }^{3,4, \dagger}$ \\ ${ }^{1}$ Institute of Applied Physics, Abbe Center of Photonics, \\ Friedrich Schiller University Jena, Albert-Einstein-Str. 15, 07745 Jena, Germany \\ ${ }^{2}$ Fraunhofer Institute for Applied Optics and Precision Engineering IOF, Albert-Einstein-Str. 7, 07745 Jena, Germany \\ ${ }^{3}$ Research School of Physics, Australian National University, Canberra ACT 2601, Australia \\ ${ }^{4}$ ARC Centre of Excellence for Transformative Meta-Optical Systems (TMOS), Australia
}

(Dated: July 7, 2021)

\begin{abstract}
We develop a concept of metasurface-assisted ghost imaging for non-local discrimination between a set of polarization objects. The specially designed metasurfaces are incorporated in the imaging system to perform parallel state transformations in general elliptical bases of quantum-entangled or classically-correlated photons. Then, only four or fewer correlation measurements between multiple metasurface outputs and a simple polarization-insensitive bucket detector after the object can allow for the identification of fully or partially transparent polarization elements and their arbitrary orientation angles. We rigorously establish that entangled photon states offer a fundamental advantage compared to classical correlations for a broad class of objects. The approach can find applications for real-time and low-light imaging across diverse spectral regions in dynamic environments.
\end{abstract}

Optical imaging of polarization properties provides a rich amount of otherwise hidden information, with applications spanning from microscopy [1] to monitoring from satellites [2]. While manipulation and measurement of polarization is conventionally performed using bulk optical elements [3], nanostructured metasurfaces allow the most flexible in-parallel polarization transformations for single-shot measurements [4], real-time imaging with a camera [5], quantum light manipulation and characterization [6-10].

Fundamental and applied interest in polarization detection at low-light illumination and across broad spectral regions, for example for bio-sensing, motivate the development of polarization ghost imaging [11]. Such schemes draw on the underlying principle originally developed for spatial ghost imaging, where the photons passing through an object are registered with a simple bucket detector $[12,13]$, while their quantum or classically-correlated pairs can be conveniently imaged at a different wavelength selected for efficient highresolution detection [14-16]. The object is characterized through multiple coincidence or correlation measurements [17] that can deliver a better signal-to-noise ratio compared to classical imaging systems, and also enable imaging with a very low number of photons $[18,19]$. However, there remains a fundamental limitation of traditional ghost polarimetry approaches due to a need for multiple reconfigurable elements such as rotating waveplates [20-27]. Yet, the unique capabilities of polarization control with metasurfaces towards potential singleshot ghost imaging configurations remains largely untapped, so far limited to the incorporation of metasurfaces for hologram generation [28].

In this Letter we present a novel concept of metasurface-assisted polarization ghost imaging. We show that by placing specially designed metasurfaces before the polarization-insensitive photon detectors, one can perform discrimination between fully or partially transparent polarization-sensitive objects within a defined set. Furthermore, in our scheme the orientation angle of each object can be simultaneously recognized from only four or fewer parallel correlation measurements. This can facilitate real-time identification of different samples for potential applications including microscopy, whereas full ghost polarimetry requires at least eight measurements with multiple time-consuming reconfigurations to determine all elements of a general Jones matrix form [25]. We also note that only discrimination of non-birefringent objects with different transversely varying transmission profiles and fixed orientations was realized previously [29].

Furthermore, we prove in a general way that ghost imaging with quantum-entangled photons offers a fundamental advantage in polarization object discrimination, compared to the use of classically polarization-correlated light. Entanglement is the most prominent feature in quantum mechanics and has become the cornerstone of rising technologies such as quantum computing [30, 31], communication [32] and metrology [33, 34]. This curious property enables unique non-local measurements since information about the properties of one particle can be obtained by performing a measurement on its entangled partner. Whereas both classical and quantum schemes can be used for imaging of non-polarizing greyscale objects [13, 35-37], this does not hold for transparent objects [9]. Importantly, the correlations between quantumentangled photons can provide additional information on the phase differences between various polarization components, which might not be detected with classical light. We rigorously derive the object properties that entail the need for entanglement.

Our proposed measurement scheme is depicted in Fig. 1. We consider a source producing a pair of probe and reference photons, which are entangled in the polar- 
ization state. Then, according to the principle of ghost imaging, only the probe photon passes through the object characterized by a polarization Jones matrix $\Omega$. We also consider a possible rotation of the object by an angle $\theta$, such that $\Omega(\theta)=R(\theta) \Omega R(-\theta)$, where $R(\theta)$ is a rotation matrix in the counterclockwise direction. The probe photon then passes through a metasurface and is registered by a polarization-insensitive click detector. The paired reference photon does not interact with the object. We place a tailored metasurface in its path, which splits the output between several polarization-insensitive detectors depending on the reference polarization state. We show in the following that by specially designing the metasurfaces, the coincidence measurements between the probe and reference at different detectors enables discrimination between polarization objects, and simultaneous identification of an arbitrary object orientation angle.

The polarization state of the photon-pairs can be defined by the density matrix

$$
\begin{array}{r}
\rho_{\text {in }}=\frac{1}{2}\left(\left|\mathrm{H}_{\mathrm{P}} \mathrm{H}_{\mathrm{R}}\right\rangle\left\langle\mathrm{H}_{\mathrm{P}} \mathrm{H}_{\mathrm{R}}|+| \mathrm{V}_{\mathrm{P}} \mathrm{V}_{\mathrm{R}}\right\rangle\left\langle\mathrm{V}_{\mathrm{P}} \mathrm{V}_{\mathrm{R}}\right|\right) \\
+q \frac{1}{2}\left(\left|\mathrm{H}_{\mathrm{P}} \mathrm{H}_{\mathrm{R}}\right\rangle\left\langle\mathrm{V}_{\mathrm{P}} \mathrm{V}_{\mathrm{R}}|+| \mathrm{V}_{\mathrm{P}} \mathrm{V}_{\mathrm{R}}\right\rangle\left\langle\mathrm{H}_{\mathrm{P}} \mathrm{H}_{\mathrm{R}}\right|\right),
\end{array}
$$

where $q$ is equal to the concurrence [38] and represents the degree of entanglement with $0 \leq q \leq 1$, from the strongest classical correlation $q=0$ to perfect entanglement $q=1$, with the Bell parameter [39] value $S=\sqrt{2}(1+q)$. Throughout this manuscript, horizontal and vertical polarization are denoted $\mathrm{H}$ and $\mathrm{V}$, respectively. The transformation of the probe photon polarization is defined by the Jones polarization transfer matrices of the object $\Omega(\theta)$ and the metasurface $M_{\mathrm{P}}$. For the reference photon, there are several outputs after the metasurface with the Jones matrices $M_{\mathrm{R}, n}$ for the different diffraction orders $n$.

After detection of the probe photon, the reduced state of the reference photon before the metasurface $M_{R}$ is determined according to the principle of remote state preparation [40] as $\rho_{\mathrm{R}}^{\prime}=\operatorname{tr}_{\mathrm{P}}\left[\left(T_{\mathrm{P}} \otimes \mathbb{1}\right) \rho_{\text {in }}\left(T_{\mathrm{P}} \otimes \mathbb{1}\right)^{\dagger}\right]$, where $\operatorname{tr}_{\mathrm{P}}(\cdot)$ is the partial trace over the probe-photon and $T_{\mathrm{P}}=M_{\mathrm{P}} \Omega(\theta)$. The normalized reduced state is then $\rho_{\mathrm{R}}=\rho_{\mathrm{R}}^{\prime} / \operatorname{tr}\left(\rho_{\mathrm{R}}^{\prime}\right)$. The expectation values of the coincidence counts between the probe and reference detectors are $\Gamma_{n}=\operatorname{tr}\left[\left(M_{\mathrm{R}, n}\right) \rho_{\mathrm{R}}\left(M_{\mathrm{R}, n}\right)^{\dagger}\right]$. We show in the following that the metasurfaces can be optimized to realize such $M_{\mathrm{P}}$ and $M_{\mathrm{R}, n}$, that every reduced state $\rho_{\mathrm{R}}$ produces a distinctive pattern formed by a collection of coincidence measurements, thereby allowing discrimination between the objects.

We determine the optimal choice of the $M_{\mathrm{P}}$ metasurface transformation for the probe photon by geometrically representing the reduced state of the reference photon $\rho_{\mathrm{R}}$ in the Poincaré sphere [41], where each $\rho_{\mathrm{R}}$ is described by a vector $\mathbf{p}=\left[p_{\mathrm{H}}, p_{\mathrm{D}}, p_{\mathrm{C}}\right]$. Here, $p_{\mathrm{H}}$ corresponds to the degree of horizontal or vertical polarization, $p_{\mathrm{D}}$ to the diagonal $[(|H\rangle+|V\rangle) / \sqrt{2}]$ or antidiagonal linear polarization at $\pm 45^{\circ}$, and $p_{\mathrm{C}}$ to the

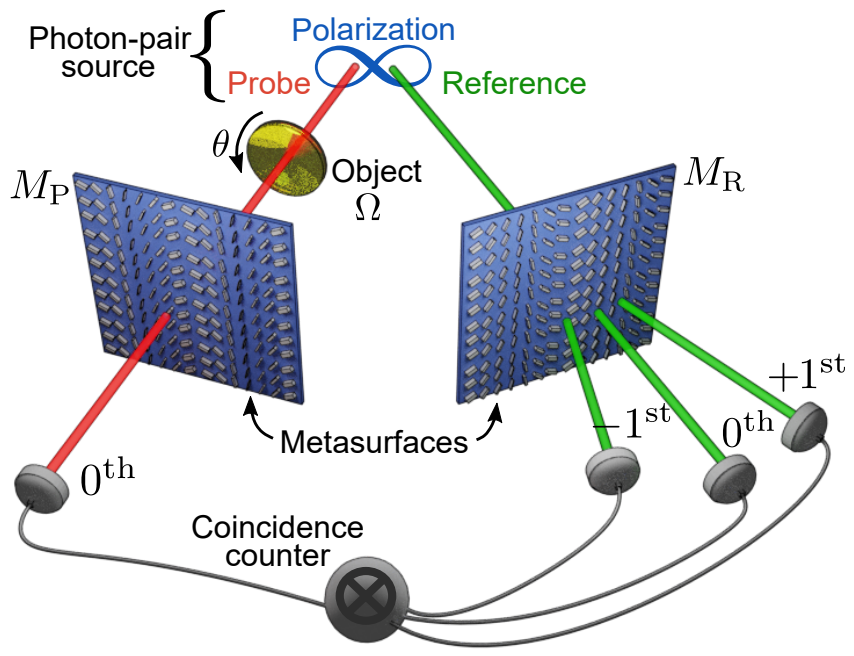

Figure 1. Sketch of the setup. Polarization transformations by metasurfaces in front of polarization-insensitive detectors enable discrimination of polarizaion objects $\Omega$ and their orientation angles $\theta$ through the coincidence measurements.

right $[(|H\rangle-i|V\rangle) / \sqrt{2}]$ or left circular polarization. The Poincare vector is found using the Pauli matrices $X, Y, Z$ with $\mathbf{p}=\left[\operatorname{tr}\left(\rho_{\mathrm{R}} Z\right), \operatorname{tr}\left(\rho_{\mathrm{R}} X\right),-\operatorname{tr}\left(\rho_{\mathrm{R}} Y\right)\right]$. If the total transformation in the probe-arm, comprising the object $\Omega$ and metasurface $M_{\mathrm{P}}$, is written in the basis of the photon-pair source as $T_{\mathrm{P}}=\sum T_{k l}|k\rangle\langle l|$ with $k, l \in\{\mathrm{H}, \mathrm{V}\}$, then

$$
\begin{aligned}
& p_{\mathrm{H}}=\left\|T_{\mathrm{P}}\right\|_{F}^{-2}\left(\left|T_{\mathrm{HH}}\right|^{2}+\left|T_{\mathrm{VH}}\right|^{2}-\left|T_{\mathrm{VV}}\right|^{2}-\left|T_{\mathrm{HV}}\right|^{2}\right) \\
& p_{\mathrm{D}}=q\left\|T_{\mathrm{P}}\right\|_{F}^{-2} \operatorname{Re}\left(T_{\mathrm{HH}} T_{\mathrm{HV}}^{*}+T_{\mathrm{VH}} T_{\mathrm{VV}}^{*}\right) \\
& p_{\mathrm{C}}=q\left\|T_{\mathrm{P}}\right\|_{F}^{-2} \operatorname{Im}\left(T_{\mathrm{HH}} T_{\mathrm{HV}}^{*}+T_{\mathrm{VH}} T_{\mathrm{VV}}^{*}\right)
\end{aligned}
$$

where $\|\cdot\|_{F}$ is the Frobenius norm. Since we require that each object produces a different reduced state, each $\rho_{\mathrm{R}}$ must occupy a unique position on or inside the Poincaré sphere.

We reveal an important relation between the components of the Poincaré vector as

$$
p_{\mathrm{H}}^{2}+\frac{p_{\mathrm{D}}^{2}+p_{\mathrm{C}}^{2}}{q^{2}}=\eta^{2}=\left(\frac{\sigma_{1 \mathrm{P}}^{2}-\sigma_{2 \mathrm{P}}^{2}}{\sigma_{1 \mathrm{P}}^{2}+\sigma_{2 \mathrm{P}}^{2}}\right)^{2},
$$

where $\sigma_{1 \mathrm{P}}$ and $\sigma_{2 \mathrm{P}}$ are the singular values of the transformation $T_{\mathrm{P}}$, see Supplementary $\mathrm{S} 1$ for the derivation. Eq. (5) indicates that the reduced state of the referencephoton $\rho_{\mathrm{R}}$ obtained from the family of transformations $T_{\mathrm{P}}$ which have the same $\eta$, will lie on an ellipsoid of revolution of long axis $\eta$ and short axis $q \eta$ given that the degree of entanglement is in the range $0 \leq q \leq 1$. This ellipsoid reduces to a point, $p_{\mathrm{H}}=p_{\mathrm{D}}=p_{\mathrm{C}}=0$, when $\sigma_{1 \mathrm{P}}=\sigma_{2 \mathrm{P}}$. Additionally, the ellipsoid is largest, for a fixed $q$, when one of the singular values is zero and $\eta=1$. For the moment, we concentrate on a scheme with a source of photon-pairs that have maximal entan- 
glement $q=1$, where the ellipsoid is a sphere with radius $\eta$.

We now use the formulated properties of the photon states to understand the need of the transformation $M_{\mathrm{P}}$ in the probe arm for object identification. To this end, we illustrate the model with a set of three objects: a transparent object (quarter-wave plate) $\Omega_{a}=|\mathrm{H}\rangle\langle\mathrm{H}|+\exp (i \pi / 2)| \mathrm{V}\rangle\langle\mathrm{V}|$ with singular values $\sigma_{1 \Omega}=\sigma_{2 \Omega}=1$, a partially transparent object $\Omega_{b}=$ $|\mathrm{H}\rangle\langle\mathrm{H}|+\exp [i(\pi / 2+0.7 i)]| \mathrm{V}\rangle\langle\mathrm{V}|$ with $\sigma_{1 \Omega}=1$ and $\sigma_{2 \Omega}=0.5$, and a fully polarizing object (horizontal polarizer) $\Omega_{c}=|\mathrm{H}\rangle\langle\mathrm{H}|$ with $\sigma_{1 \Omega}=1$ and $\sigma_{2 \Omega}=0$. These definitions are for an object rotation angle $\theta=0$. Since rotation is a unitary transformation, the mentioned singular values of each object remain unaffected and the associated reference-photon reduced states lie on a sphere with a radius defined by Eq. (5) for all object angles. Since the considered objects have a rotation symmetry of $\pi$, we aim to have different $\rho_{\mathrm{R}}$ in the interval $0 \leq \theta<\pi$ for the angle identification.

We illustrate the effect of metasurface transformation $M_{\mathrm{P}}$ on the discrimination of objects in Fig. 2. We compare the cases of no metasurface with $M_{\mathrm{P}}=I$ in the left, non-optimal transformation in the middle, and optimized transformation in the right column. In the top row, we show in the Poincaré sphere the two orthonormal right singular vectors of $M_{\mathrm{P}}, \mathbf{m}_{j \mathrm{P}}$ with $j \in\{1,2\}$ (see Supplementary S2), whose magnitude has been scaled with their corresponding singular value $\sigma_{j \mathrm{P}}$. The rows (a-c) represent the normalized reduced states of the probe photon for the three chosen objects $\Omega_{a, b, c}$, respectively.

For the simplest scenario without a metasurface $M_{\mathrm{P}}$ in the probe arm presented in Fig. 2(left column), a fully transparent wave-plate $\Omega_{a}$ always corresponds to a single point, such that its orientation angle cannot be identified. Indeed, in this case $T_{\mathrm{P}}=\Omega$ with singular values $\sigma_{\mathrm{P}}=\sigma_{\Omega}$, and according to Eq. (5) any transparent phase object with $\sigma_{1 \Omega}=\sigma_{2 \Omega}$ will generate reduced states $\rho_{\mathrm{R}}$ that stay on the origin. Hence, discrimination between transparent phase objects and determination of their rotations cannot be performed without a metasurface $M_{\mathrm{P}}$ acting on the polarization of the probe photon before a polarizationinsensitive detector.

In Fig. 2(middle column), we illustrate a case of fully polarizing transformation $M_{\mathrm{P}}$ with $\sigma_{2 \mathrm{P}}=0$, such that only one polarization is transmitted. This represents a quantum projection measurement for the state $\mathbf{m}_{1 \mathrm{P}}$. We note that if an object $\Omega$ and/or $M_{\mathrm{P}}$ have one singular value equal to zero, then its product, $T_{\mathrm{P}}=M_{\mathrm{P}} \Omega$, also has one null singular value. This causes, according to Eq. (5), the reduced state of the reference-photon to lie on the surface of the Poincaré sphere. Consequently, not all objects and rotation angles can be distinguished since the closed loops formed by the objects' reduced states would cross.

To enable discrimination in the general case, the ratio

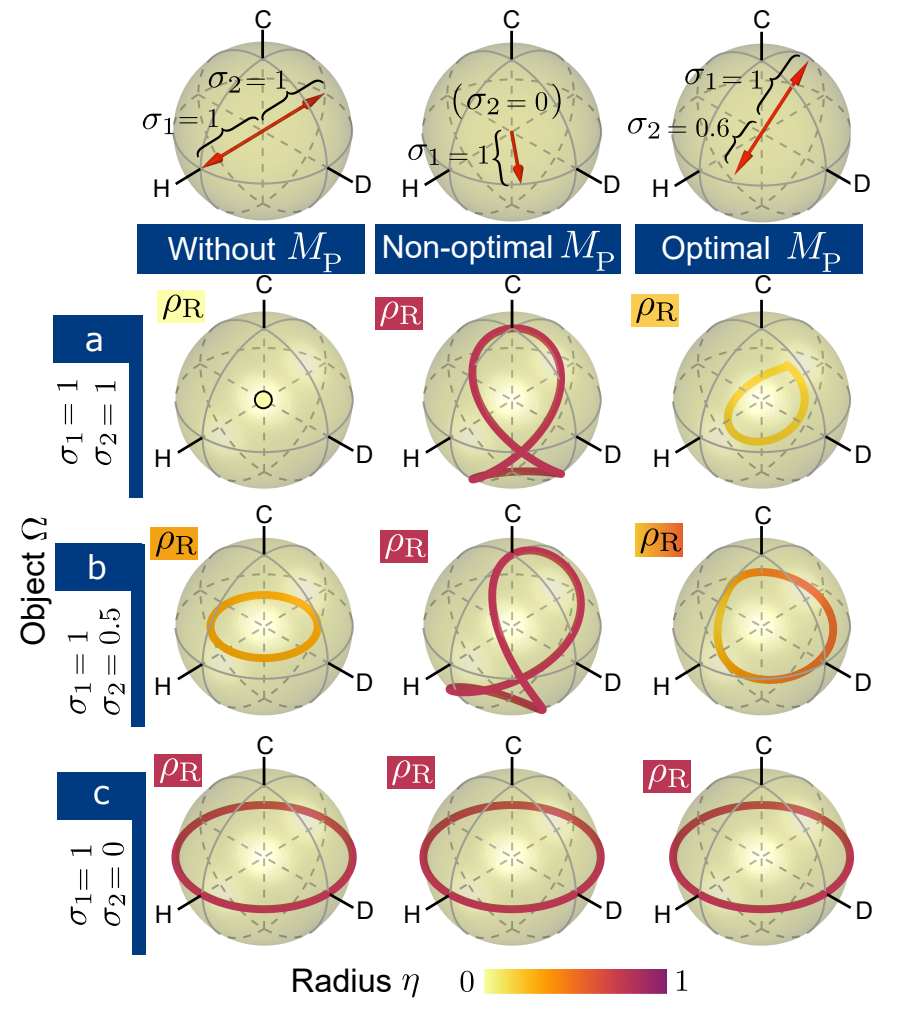

Figure 2. Metasurface transformation $M_{\mathrm{P}}$ enabling the object discrimination. Top: The singular eigenvectors of $M_{\mathrm{P}}$, scaled by the corresponding singular values, represented in the Poincaré sphere for the cases of (left) no metasurface, (middle) fully polarizing transformation, (right) optimal partially polarizing case. The axes of the Poincaré sphere correspond to horizontal (H), diagonal (D) and right circular (C) polarizations. (a-c) Reduced states of the reference-photon after measuring the probe-photon $\rho_{\mathrm{R}}$ produced by a set of three objects $\Omega_{a, b, c}$, for different $M_{\mathrm{P}}$ in each column. The curves are formed from the points corresponding to different object rotation angles in the range $0 \leq \theta<\pi$.

of the smaller to larger singular values of $M_{\mathrm{P}}$ has to be in the range $0<\sigma_{2} / \sigma_{1}<1$, i.e., it should be a partially polarizing transformation. Additionally, its largest singular value should be close to 1 to ensure high photon counts. The reduced states for a numerically optimized polarization basis of $M_{\mathrm{P}}$ are shown in Fig. 2(right column). They do not cross and enable discrimination, confirming the above analysis of the required singular values. The displayed elliptical polarization basis is formed by the two orthonormal right singular vectors of $M_{\mathrm{P}}$, represented by arrows in the top-row plot. Importantly, the optimal $M_{\mathrm{P}}$ depends on the given set of objects.

Next, we discuss conditions on the optimal polarization bases of the transformation $M_{\mathrm{R}}$ in the reference arm, which ensures that each object $\Omega$ has a unique pattern in the coincidences. To achieve practically relevant solutions, we impose two additional conditions: object identification must be achieved using a small number of outputs and the total number of photon counts in all outputs 
should be larger than zero for any object. We note that a specially designed metasurface with four diffraction outputs can facilitate a full characterization of single-photon polarization [7], thereby allowing for object discrimination based on the different reference photon states $\rho_{R}$. We find that discrimination between particular sets of objects may be performed with a smaller set of outputs, which can simplify the measurements and improve signalto-noise ratio since photon counts are concentrated in fewer outputs. The optimal design that meets these requirements can be investigated by writing the expectation value of the coincidence counts with the $n$-th reference output as (see Supplementary S2)

$$
\begin{aligned}
\Gamma_{n}= & \frac{1}{2}\left(\sigma_{1 \mathrm{R}, n}^{2}+\sigma_{2 \mathrm{R}, n}^{2}\right) \\
& +\frac{1}{2}\left(\mathbf{p} \cdot \mathbf{m}_{1 \mathrm{R}, n}\right)\left(\sigma_{1 \mathrm{R}, n}^{2}-\sigma_{2 \mathrm{R}, n}^{2}\right),
\end{aligned}
$$

where $\sigma_{\mathrm{R}, n}$ stands for a singular value of the transformation $M_{\mathrm{R}, n}$, and $\mathbf{m}_{\mathrm{R}, n}$ is its right singular vector represented in the Poincaré sphere. Additionally, the output pattern in coincidences can be represented in a space $\mathcal{D}$ such that each expectation value $\Gamma_{n}$ corresponds to a coordinate along the $n$-th dimension and each pattern in coincidences is mapped as a point in $\mathcal{D}$.

One approach to fulfill the requirement of non-zero photon counts consists of having equal singular values for all outputs of the metasurface $M_{\mathrm{R}}, \sigma_{1 \mathrm{R}, n}=\sigma_{1 \mathrm{R}}$ and $\sigma_{2 \mathrm{R}, n}=\sigma_{2 \mathrm{R}}$, and also that the sum of their right singular vectors to be equal to zero, $\sum \mathbf{m}_{1 \mathrm{R}, n}=0$. Thus, $\sum \Gamma_{n}=(1 / 2) \sum\left(\sigma_{1 \mathrm{R}}^{2}+\sigma_{2 \mathrm{R}}^{2}\right)$ is a constant different from zero, meaning that the photon counts will be non-zero. If we consider $M_{\mathrm{R}}$ with three outputs, the condition $\sum \mathbf{m}_{1 \mathrm{R}, n}=0$ would indicate that each pair of vectors $\mathbf{m}$ encloses an angle of $120^{\circ}$ and they would all lie in one plane in the Poincaré sphere. This plane has to be chosen such that each object $\Omega$ has a unique pattern in the coincidences. To demonstrate this, we return to our example with objects $\Omega_{a, b, c}$.

The reduced states $\rho_{\mathrm{R}}$ from the objects $\Omega(\theta)$ and the optimal $M_{\mathrm{P}}$ discussed in Fig. 2 are depicted together in the Poincaré sphere of Fig. 3(a). A plane that can encompass the three vectors $\mathbf{m}$ while allowing for separation of the projected reduced states is illustrated in blue along with its normal $\mathbf{N}$. The projections of all the reduced states $\rho_{\mathrm{R}}$ into this plane are well separated, as shown in Fig. 3(b). They can then be mapped into the space $\mathcal{D}$ of the coincidences at the outputs using the three projection vectors of $M_{\mathrm{R}}$ depicted in Fig. 3(c), as described above. The direction of the optimal vector $\mathbf{N}$, and therefore the plane where the vectors $\mathbf{m}_{1 \mathrm{R}, n}$ lie can be found numerically. For the set of objects investigated here, at least three outputs are needed to achieve discrimination. Two or four outputs may be needed for other sets of objects (see Supplementary S5 for an example).

The optimal form of $M_{\mathrm{P}}$ and $M_{\mathrm{R}}$ in each arm of the setup can be found numerically. We note that the po- larization transformations in principle can also be implemented with a collection of bulk optical elements; however, at the expense of complex designs sensitive to alignment. Here we show that these polarization manipulations can be realized using nanostructured dielectric metasurfaces, which can effectively act as partial polarizers in arbitrary elliptical bases with any required extinction ratio [7, 42]. Each metasurface is a flat optical element composed of a periodically repeated array of nano-resonators called meta-gratings. We perform optimization of the phase retardances and orientations associated with the individual nano-resonators to realize the required transformations, see details in Supplementary S4 and S6. Specifically, we design a metasurface for the probe arm such that its zeroth diffraction order realizes $M_{\mathrm{P}}$. Similarly, a second metasurface is placed in the reference arm where the three outputs of $M_{\mathrm{R}}$ correspond to three diffraction orders, as illustrated in Fig. 1. The unit-cell geometries of numerically found optimal metagratings for the considered set of objects $\Omega_{a, b, c}$ are depicted in Fig. 3(d).

The main result of this Letter is shown in Fig. 3(e), where coincidence patterns at three diffraction orders for the objects $\Omega_{a, b, c}(\theta)$ are represented in the space $\mathcal{D}=\left\{\Gamma_{-1}, \Gamma_{0}, \Gamma_{+1}\right\}$, where each point on the plotted curves corresponds to a different object rotation angle $\theta$. The inset shows an example for the polarizing object $\Omega_{c}$ at $\theta=0$. This showcases that the full identification of objects with their corresponding rotation angle is possible by optimizing $M_{\mathrm{P}}$ and $M_{\mathrm{R}}$. As designed, the chosen three outputs of metasurface $M_{\mathrm{R}}$ map the geometric shape of Fig. 3(b) into the space $\mathcal{D}$ in such a way that all the coincidences correspond to practically relevant non-zero photon counts.

After demonstrating that objects and their rotation angles can be discriminated using entangled states of light, next we show that entanglement is necessary to distinguish specific classes of objects. To this end, we explore the effect of reducing the entanglement until reaching only classical correlation, $q=0$. We consider a different set of polarization-sensitive objects that contains only generalized retarders $\Omega=|\mathrm{H}\rangle\langle\mathrm{H}|+\exp (i \phi)| \mathrm{V}\rangle\langle\mathrm{V}|$, each with its own phase difference $\phi$ between the horizontal and vertical polarization. Here, we do not seek to identify the rotation of each of them but just discern them based on $\phi$.

The optimal $M_{\mathrm{P}}$ necessary to discern these phase objects for any value of $q$ is a diagonal polarizer. Notice here that this element can obviously be implemented simply using a conventional polarizer instead of a metasurface. The corresponding reduced states $\rho_{\mathrm{R}}$ of this set of phase objects are depicted in Fig. 4 for different levels of entanglement $q$. As can be seen, this chosen set of phase objects is a quite peculiar one because their reduced states have the same $p_{\mathrm{H}}$ Poincaré vector component [see Eq. (2)], as $p_{\mathrm{H}}$ does not depend on the phase $\phi$. 


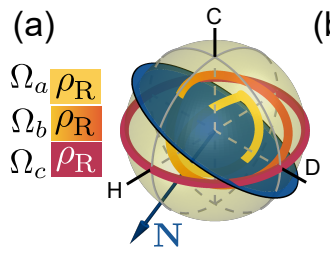

(d)

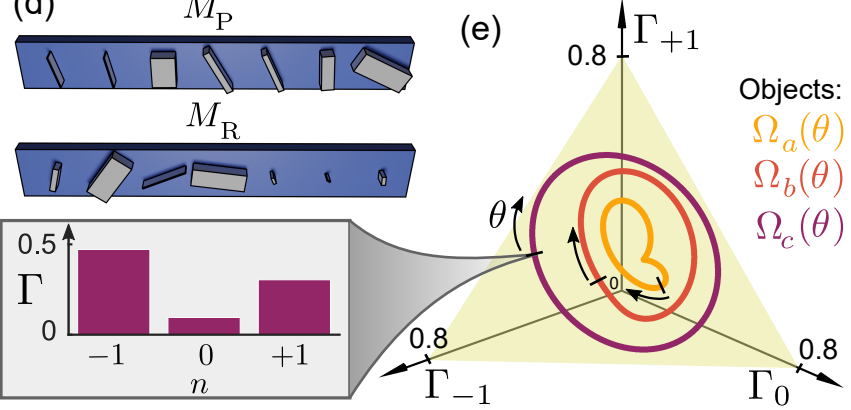

(b) $\quad$ D $\quad$ (c)

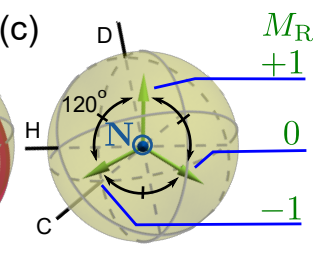

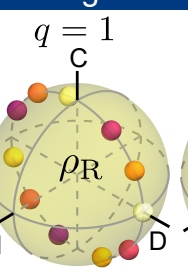
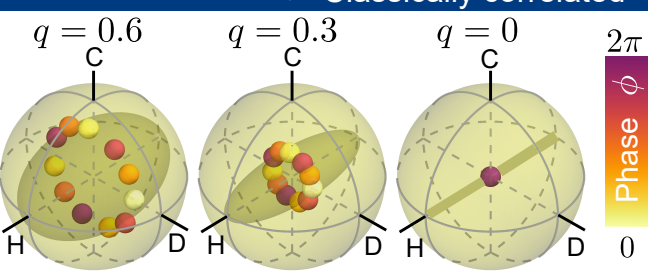

Figure 4. Reduced state of the reference-photon after measuring the probe-photon $\rho_{\mathrm{R}}$ at different levels of entanglement. Here, the probe-photon interacts with a set of different fully transmissive phase objects, $\Omega=|\mathrm{H}\rangle\langle\mathrm{H}|+\exp (i \phi)| \mathrm{V}\rangle\langle\mathrm{V}|$ (phase $\phi$ shown in the color scale), one at a time, and also with an optimal $M_{\mathrm{P}}$ that acts as a diagonal polarizer.

between probe and reference-photons are needed to enable discrimination of all types of objects. We believe that these fundamental results can stimulate the practical development of efficient and integrated optical schemes for characterization of objects with polarizationsensitive transmission characteristics across a broad spectral range. In particular, these can benefit applications demanding accurate discrimination of objects with various polarization characteristics, including sets of phase objects like biological samples.

The authors thank Michael Brodsky, Kai Wang, Shaun Lung, Jihua Zhang for insightful discussions and comments. This work was supported by the Thuringian Ministry for Economy, Science, and Digital Society (2021 FGI 0043), the German Federal Ministry of Education and Research (FKZ 13N14877), the German Research Foundation (IRTG 2675), the European Union through the ERASMUS+ program, the German Academic Exchange Service (grant 57388353), and UA-DAAD exchange scheme. A.A.S. also acknowledges the support by the Australian Research Council (DP160100619, DP190101559, CE200100010) and US AOARD (19IOA053). $\theta$, their Poincaré component $p_{\mathrm{H}}$ becomes dependent on the phase $\phi$ and they could be identified using only classical correlations (see Supplementary S3). In a nutshell, transformations $T_{\mathrm{P}}$ that have the same $p_{\mathrm{H}}$ component can only be discriminated if the photon-pair source has a high level of entanglement. Our conclusion on the advantage of entanglement generally agrees with the results of Ref. [9], where images of polarization-dependent patterns imprinted with a metasurface had higher visibility across different orientation angles for larger Bell parameter values.

In summary, we proposed a general approach for ghost discrimination between polarization-sensitive objects and simultaneous identification of their rotation through nonlocal measurements using non-classical light. This is achieved by using nanostructured metasurfaces designed to perform tailored polarization transformations. Importantly, we also proved that non-classical correlations
* andres.vega@uni-jena.de

† andrey.sukhorukov@anu.edu.au

[1] N.-J. Jan, J. L. Grimm, H. Tran, K. L. Lathrop, G. Wollstein, R. A. Bilonick, H. Ishikawa, L. Kagemann, J. S. Schuman, and I. A. Sigal, Polarization microscopy for characterizing fiber orientation of ocular tissues, Biomed. Opt. Express 6, 4705 (2015).

[2] A. Puthukkudy, J. V. Martins, L. A. Remer, X. G. Xu, O. Dubovik, P. Litvinov, B. McBride, S. Burton, and H. M. J. Barbosa, Retrieval of aerosol properties from airborne hyper-angular rainbow polarimeter (airharp) observations during acepol 2017, Atmos. Meas. Tech. 13, 5207 (2020).

[3] M. Chekhova and P. Banzer, Polarization of Light In Classical, Quantum, and Nonlinear Optics (De Gruyter, Berlin, 2021). 
[4] A. Martinez, Polarimetry enabled by nanophotonics, Science 362, 750 (2018).

[5] N. A. Rubin, G. D'Aversa, P. Chevalier, Z. J. Shi, W. T. Chen, and F. Capasso, Matrix Fourier optics enables a compact full-Stokes polarization camera, Science $\mathbf{3 6 5}$, eaax1839 (2019).

[6] T. Stav, A. Faerman, E. Maguid, D. Oren, V. Kleiner, E. Hasman, and M. Segev, Quantum entanglement of the spin and orbital angular momentum of photons using metamaterials, Science 361, 1101 (2018).

[7] K. Wang, J. G. Titchener, S. S. Kruk, L. Xu, H. P. Chung, M. Parry, I. I. Kravchenko, Y. H. Chen, A. S. Solntsev, Y. S. Kivshar, D. N. Neshev, and A. A. Sukhorukov, Quantum metasurface for multiphoton interference and state reconstruction, Science 361, 1104 (2018).

[8] P. Georgi, M. Massaro, K. H. Luo, B. Sain, N. Montaut, H. Herrmann, T. Weiss, G. X. Li, C. Silberhorn, and T. Zentgraf, Metasurface interferometry toward quantum sensors, Light Sci. Appl. 8, 70 (2019).

[9] C. Altuzarra, A. Lyons, G. H. Yuan, C. Simpson, T. Roger, J. S. Ben-Benjamin, and D. Faccio, Imaging of polarization-sensitive metasurfaces with quantum entanglement, Phys. Rev. A 99, 020101 (2019).

[10] A. S. Solntsev, G. S. Agarwal, and Y. S. Kivshar, Metasurfaces for Quantum Photonics, Nat. Photon. 15, 327 (2021).

[11] H. Kellock, T. Setala, A. T. Friberg, and T. Shirai, Polarimetry by classical ghost diffraction, J. Opt. 16, 055702 (2014).

[12] T. B. Pittman, Y. H. Shih, D. V. Strekalov, and A. V. Sergienko, Optical imaging by means of 2-photon quantum entanglement, Phys. Rev. A 52, R3429 (1995).

[13] A. Valencia, G. Scarcelli, M. D'Angelo, and Y. Shih, Two-photon imaging with thermal light, Phys. Rev. Lett. 94, 063601 (2005).

[14] K. W. C. Chan, M. N. O'Sullivan, and R. W. Boyd, Twocolor ghost imaging, Phys. Rev. A 79, 033808 (2009).

[15] S. Karmakar and Y. H. Shih, Two-color ghost imaging with enhanced angular resolving power, Phys. Rev. A 81, 033845 (2010).

[16] R. S. Aspden, N. R. Gemmell, P. A. Morris, D. S. Tasca, L. Mertens, M. G. Tanner, R. A. Kirkwood, A. Ruggeri, A. Tosi, R. W. Boyd, G. S. Buller, R. H. Hadfield, and M. J. Padgett, Photon-sparse microscopy: visible light imaging using infrared illumination, Optica 2, 1049 (2015).

[17] B. I. Erkmen and J. H. Shapiro, Ghost imaging: from quantum to classical to computational, Adv. Opt. Photon. 2, 405 (2010).

[18] G. Brida, M. Genovese, and I. R. Berchera, Experimental realization of sub-shot-noise quantum imaging, Nat. Photon. 4, 227 (2010).

[19] P. A. Morris, R. S. Aspden, J. E. C. Bell, R. W. Boyd, and M. J. Padgett, Imaging with a small number of photons, Nat. Commun. 6, 5913 (2015).

[20] A. Hannonen, A. T. Friberg, and T. Setala, Classical spectral ghost ellipsometry, Opt. Lett. 41, 4943 (2016).

[21] A. Hannonen, A. T. Friberg, and T. Setala, Classical ghost-imaging spectral ellipsometer, J. Opt. Soc. Am. A 34, 1360 (2017).

[22] P. Janassek, S. Blumenstein, and W. Elsasser, Recovering a hidden polarization by ghost polarimetry, Opt. Lett. 43, 883 (2018).

[23] D. F. Shi, J. M. Zhang, J. Huang, Y. J. Wang,
K. Yuan, K. F. Cao, C. B. Xie, D. Liu, and W. Y. Zhu, Polarization-multiplexing ghost imaging, Opt. Lasers Eng. 102, 100 (2018).

[24] A. S. Chirkin, P. P. Gostev, D. P. Agapov, and S. A. Magnitskiy, Ghost polarimetry: ghost imaging of polarization-sensitive objects, Laser Phys. Lett. 15, 115404 (2018).

[25] A. Hannonen, B. J. Hoenders, W. Elsasser, A. T. Friberg, and T. Setala, Ghost polarimetry using Stokes correlations, J. Opt. Soc. Am. A 37, 714 (2020).

[26] M. Rosskopf, T. Mohr, and W. Elsasser, Ghost polarization communication, Phys. Rev. Appl. 13, 034062 (2020).

[27] S. Magnitskiy, D. Agapov, and A. Chirkin, Ghost polarimetry with unpolarized pseudo-thermal light, Opt. Lett. 45, 3641 (2020).

[28] H. C. Liu, B. A. Yang, Q. H. Guo, J. H. Shi, C. Y. Guan, G. X. Zheng, H. Muhlenbernd, G. X. Li, T. Zentgraf, and S. Zhang, Single-pixel computational ghost imaging with helicity-dependent metasurface hologram, Sci. Adv. 3, e1701477 (2017).

[29] M. Malik, H. Shin, M. O'Sullivan, P. Zerom, and R. W. Boyd, Quantum ghost image identification with correlated photon pairs, Phys. Rev. Lett. 104, 163602 (2010).

[30] S. Slussarenko and G. J. Pryde, Photonic quantum information processing: A concise review, Appl. Phys. Rev. 6, 041303 (2019).

[31] H. L. Huang, D. C. Wu, D. J. Fan, and X. B. Zhu, Superconducting quantum computing: a review, Sci. ChinaInf. Sci. 63, 180501 (2020).

[32] N. Gisin and R. Thew, Quantum communication, Nat. Photon. 1, 165 (2007).

[33] V. Giovannetti, S. Lloyd, and L. Maccone, Advances in quantum metrology, Nat. Photon. 5, 222 (2011).

[34] L. Pezze, A. Smerzi, M. K. Oberthaler, R. Schmied, and P. Treutlein, Quantum metrology with nonclassical states of atomic ensembles, Rev. Mod. Phys. 90, 035005 (2018).

[35] J. H. Shapiro, D. Venkatraman, and F. N. C. Wong, Classical imaging with undetected photons, Sci. Rep. 5, 10329 (2015).

[36] R. S. Bennink, S. J. Bentley, and R. W. Boyd, "twophoton" coincidence imaging with a classical source, Phys. Rev. Lett. 89, 113601 (2002).

[37] A. Gatti, E. Brambilla, M. Bache, and L. A. Lugiato, Ghost imaging with thermal light: Comparing entanglement and classical correlation, Phys. Rev. Lett. 93, 093602 (2004).

[38] W. K. Wootters, Entanglement of formation of an arbitrary state of two qubits, Phys. Rev. Lett. 80, 2245 (1998).

[39] A. Aspect, P. Grangier, and G. Roger, Experimental realization of einstein-podolsky-rosen-bohm gedankenexperiment - a new violation of Bell inequalities, Phys. Rev. Lett. 49, 91 (1982).

[40] N. A. Peters, J. T. Barreiro, M. E. Goggin, T. C. Wei, and P. G. Kwiat, Remote state preparation: Arbitrary remote control of photon polarization, Phys. Rev. Lett. 94, 150502 (2005).

[41] A. Z. Goldberg, P. De La Hoz, G. Bjork, A. B. Klimov, M. Grassl, G. Leuchs, and L. L. Sanchez-Soro, Quantum concepts in optical polarization, Adv. Opt. Photon. 13, 1 (2021).

[42] S. Lung, K. Wang, K. Z. Kamali, J. H. Zhang, M. Rahmani, D. N. Neshev, and A. A. Sukhorukov, Complex-birefringent dielectric metasurfaces for arbi- 
trary polarization-pair transformations, ACS Photonics 7, 3015 (2020). 